\title{
Carcinomatous metastasis to the vertebral bodies
}

\author{
R. A. B. DRURY, P. H. PALMER, AND WILMA J. HIGHMAN \\ From the Central Middlesex Hospital, London
}

SYNOPSIS The spread of carcinoma to the vertebral bodies has been studied by the long vertebral $\vec{\circ}_{-}^{\circ}$ slice technique. Vertebral secondaries were found in one third of all patients with carcinoma, and $\vec{\omega}$ in almost one half of those dying with distant metastases. Haematogenous dissemination and $\frac{\mathrm{T}}{\circ}$ direct lymphatic spread both appeared to produce vertebral metastases. Radiology and less effective? investigation of the skeleton at necropsy underestimate the incidence of bony metastases. This. $\vec{v}$ method is recommended as an easy and rapid one of examining the bone marrow, and gives $a_{+}^{+}$ good indication of the presence of metastases in the whole of the skeletal system.

The examination of the skeleton is one of the most difficult aspects of necropsy technique. Methods, and their results, vary widely, and the bone marrow is commonly investigated less thoroughly than any other system. Of necessity the direct inspection of individual bones must be limited, and few patients can have a complete radiological survey of the skeleton before or after death. The removal of a long slice from the front of the vertebral bodies with a chisel in a series of 573 patients with carcinoma has demonstrated a high incidence of vertebral metastases. This method is quick and simple, and has produced unexpected information in so many necropsies that it is recommended as a valuable routine procedure for the general examination of the bone marrow.

\section{MATERIAL AND METHODS}

Necropsies were performed in the Central Middlesex Hospital on 1,775 consecutive adult patients over 20 years of age between January 1960 and September 1962; there were 919 males and 856 females. These represented approximately $70 \%$ of all patients dying in a regional acute general hospital. The material was unselected except for the almost complete exclusion of coroner's necropsies, but nearly all of these subjects were known to have died of diseases other than carcinoma, and it is believed that the carcinoma patients represented a reasonably complete and unbiased group. The age and sex distribution of all the patients, and those with carcinoma, is shown in Figure 1.

Necropsy was periormed in each case; the trachea and mediastinal structures, including the thoracic aorta, were removed with the thoracic organs; the abdominal aorta and vena cava were excised with the kidneys. This

Received for publication 27 September 1963. exposed the whole length of the cervical, dorsal, and lumbar vertebral bodies, and a complete coronal slice_was removed in one piece from the fronts of the vertebrale bodies with a 2 in. $(5 \mathrm{~cm}$.) carpenter's chisel. (This cost $₫$ about 7s. 6d. from an ironmonger; a metal ferrule around the end of the wooden handle was an advantage.) The

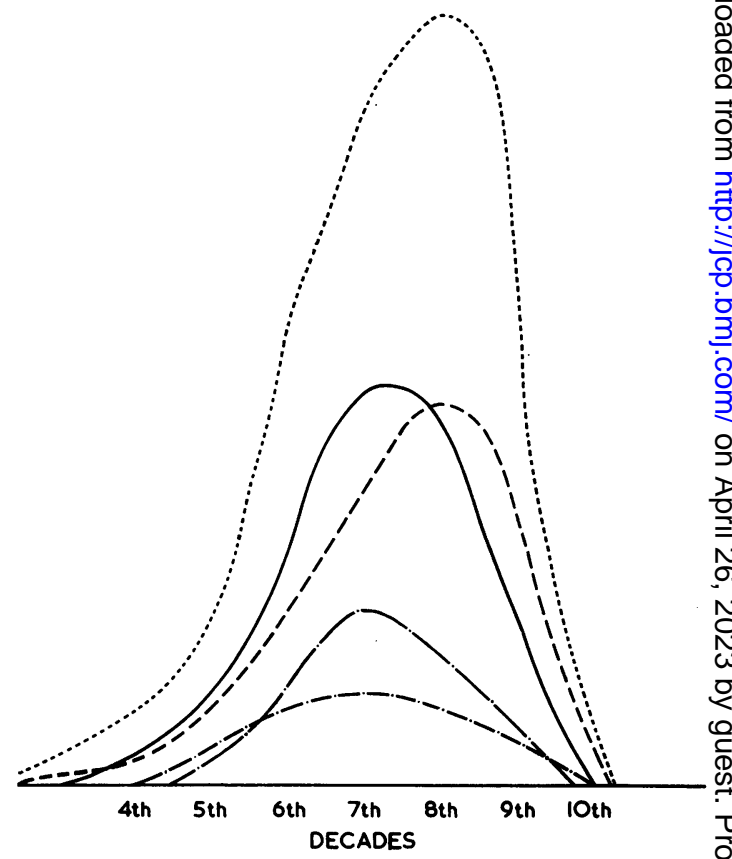

FIG. 1. Age distribution (in decades) of all patients examined, with sex distribution and incidence of carcinoma $\overrightarrow{\mathrm{D}}$ ...... all patients _- - males with carcinoma all males -. - females with carcinoma 


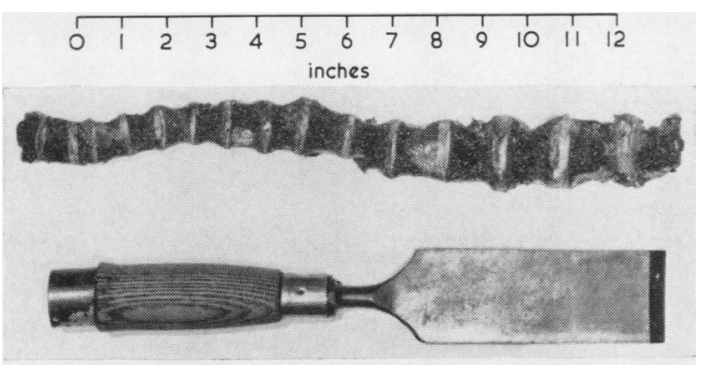

FIG. 2. The long vertebral slice, showing focal metastases (from breast) and collapse of an upper dorsal vertebral body.
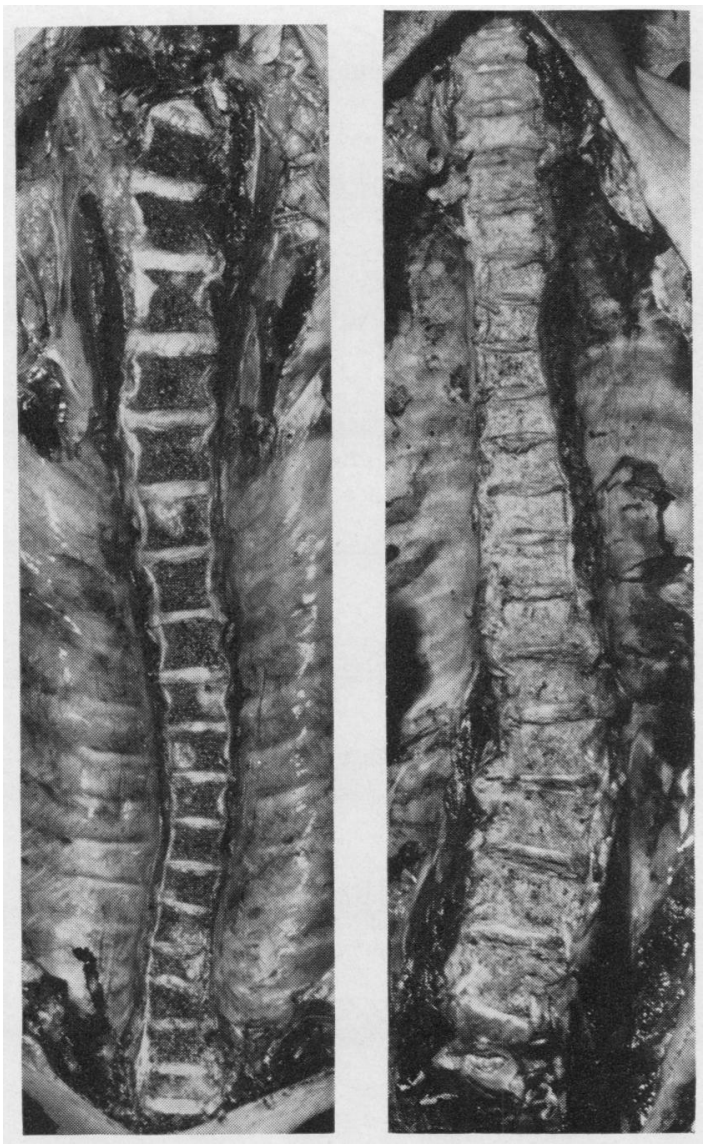

FIG. 3. The vertebral bodies exposed by the long slice. Left: scattered metastases (from breast). Right: diffuse carcinomatous replacement of all dorsal and lumbar vertebral bodies (from prostate). chisel was used with the bevel downwards and was struck with a mallet, starting at the fifth lumbar vertebra and working upwards; when the lower dorsal vertebrae were reached the curvature of the spine made it difficult to keep the slice parallel to the front of the vertebral bodies, and it was now easier to withdraw the chisel and to start again, working from the lower cervical vertebrae downwards until this cut joined up with the lower cut. A slice approximately $40 \mathrm{~cm}$. long, $1 \mathrm{~cm}$. thick, and averaging $3 \mathrm{~cm}$. in width came away (Fig. 2). This was suitable for photography and histology and required no cleaning as there was no bone dust covering the bone marrow. The body was not mutilated and there was no need for special reconstruction, as is necessary after the removal of a long bone. The technique is rapid and can be carried out by a post-mortem room attendant in less than one minute. The complete absence of bone dust made interpretation of the chisel slice far easier than that of a wedge sawn from the lumbar vertebrae; the colour and consistency of the bone marrow were well preserved, and individual bony trabeculae could be seen. It was found that in the majority of cases the presence or absence of vertebral metastases was clear from macroscopic examination (Fig. 3), though inexperienced pathologists tended to mistake patches of fatty marrow (common in the aged), old fractures, Schmorl's nodes, or fibrous scars for secondary carcinomata. In a few instances, diffuse carcinomatous infiltration of the bone marrow due to oat cell carcinoma of the bronchus produced very little macroscopic abnormality. Histological sections were examined routinely from selected areas from the vertebral slice, whether metastases were seen macroscopically or not; a small number of false negatives seem to be unavoidable on naked-eye examination, but false positives were infrequent after experience with this method. Specimens were fixed in formalin and decalcified with $5 \%$ formic acid; cellular detail was well preserved and there was no significant crushing or distortion of marrow structure.

In addition to the vetrebral slice, the chisel may be used to remove the whole of some vertebral bodies in order to expose localized areas of the spinal cord. This quickly produced specimens without the necessity of turning the body over, and was useful if only part of the spinal cord required examination.

\section{FINDINGS}

The 1,775 necropsies included 575 patients with carcinoma $(32 \%)$. Cases of primary bone marrow disease (leukaemia, myeloma, etc.), glioma, and all sarcomas, including lymphoid tumours, were excluded. Microscopic carcinomata found by subsequent histology (usually in the prostate) were not included. In most cases the primary tumour had been diagnosed during life though in some the primary site was in error or in doubt; in a smaller number the carcinoma was an unexpected finding, and if this tumour was macroscopically visible it was included in the series even though it might have 


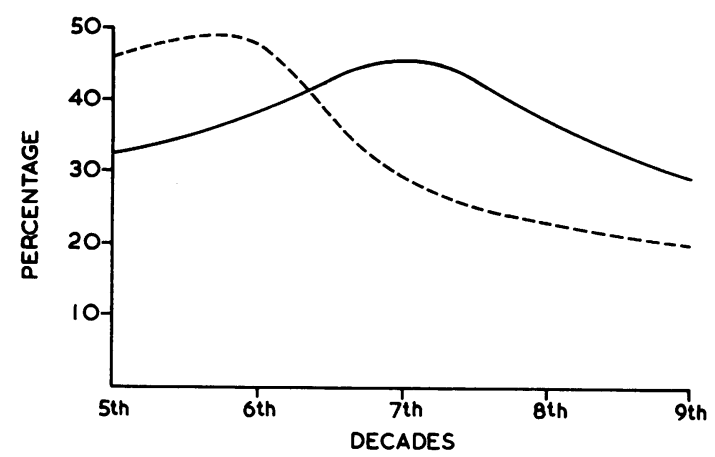

FIG. 4. Percentage incidence of all carcinomas in all males (-) and in all females (- - - -).

played no direct part in the final illness. There were 330 men and 245 women with carcinoma; their age distribution is shown in Figure 1. In men, the incidence of carcinoma was reasonably constant throughout all decades (approximately 30 to $40 \%$ ), and did not diminish greatly with old age, but in women there was a peak incidence of carcinoma in the sixth decade, with a steadily falling incidence in subsequent decades (Fig. 4). The commonest primary site of carcinoma was lung (190 patients, including bronchus, lung, and pleura), followed by intestine (73), breast (69), and stomach (66); the primary sites, with the sex distribution, are shown in Figure 5. One third of the carcinomata arose from lung, and whilst at first sight this appears to be an excessive proportion in comparison with the Registrar General's (1963) figure of between 20 and
$25 \%$ the national ante-mortem/post-mortem certi- $\frac{0}{\vec{*}}$ fication survey showed that carcinoma of the lung. was still $18 \%$ under-diagnosed (Heasman, 1962); $; \overrightarrow{\vec{F}}$ our high proportion of carcinoma of the lung maynot, therefore, be due to selection or over-diagnosis. There were 117 patients with carcinoma without $\frac{\bar{c}}{\bar{p}}$ metastases of any kind, and an additional 54 with $\vec{\nabla}$ lymph node metastases only; this left 404 with distant metastases, mainly haematogenous.

Secondary carcinoma, proven by histology, was $\vec{\circ}$ found in one or more vertebral bodies in 192 of the 575 patients with carcinoma (Fig. 6). The overall $\vec{\rho}$ incidence of histologically proven vertebral meta- $\frac{-}{\circ}$ stasis was $33.4 \%$ (males $31 \%$, females $36 \%$ ). Of the? 454 patients with lymphatic and haematogenous. metastases, $42 \%$ had vertebral secondaries, and this figure is raised to $47.5 \%$ in the 404 patients with distant visceral metastases. Thus it was found that $\circ$ one third of all patients with carcinoma had vertebral metastases, and these were present in $\bar{c}$ almost one half of the patients dying with distant metastases.

The primary carcinoma with the highest percentage of vertebral metastases was breast $(74 \%)$. Figure 7 shows the percentage of histologically proven vertebral metastases according to primary sites, and in addition to breast, prostate $(65 \%)$ and thyroid $(60 \%)$, in which a high incidence of bony $\frac{2}{8}$ secondaries was expected, surprisingly high figures $\unrhd$ were found in carcinoma of the uterine cervix $(50 \%)$, $\overrightarrow{0}$ bladder $(42 \%)$, and pancreas $(17 \%)$. Even intestinal 3 carcinoma, usually still confined to the abdomen showed vertebral metastases in $8 \%$. Carcinoma of

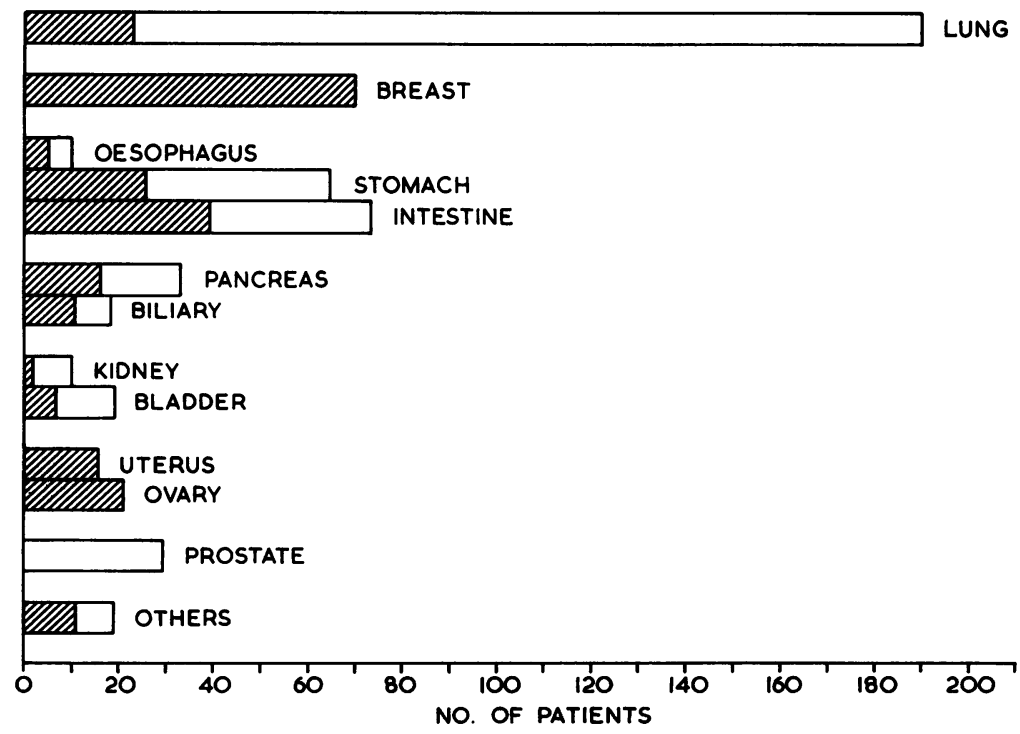

FIG. 5. The primary sites of the carcinomas. Crosshatched $=$ all females. 


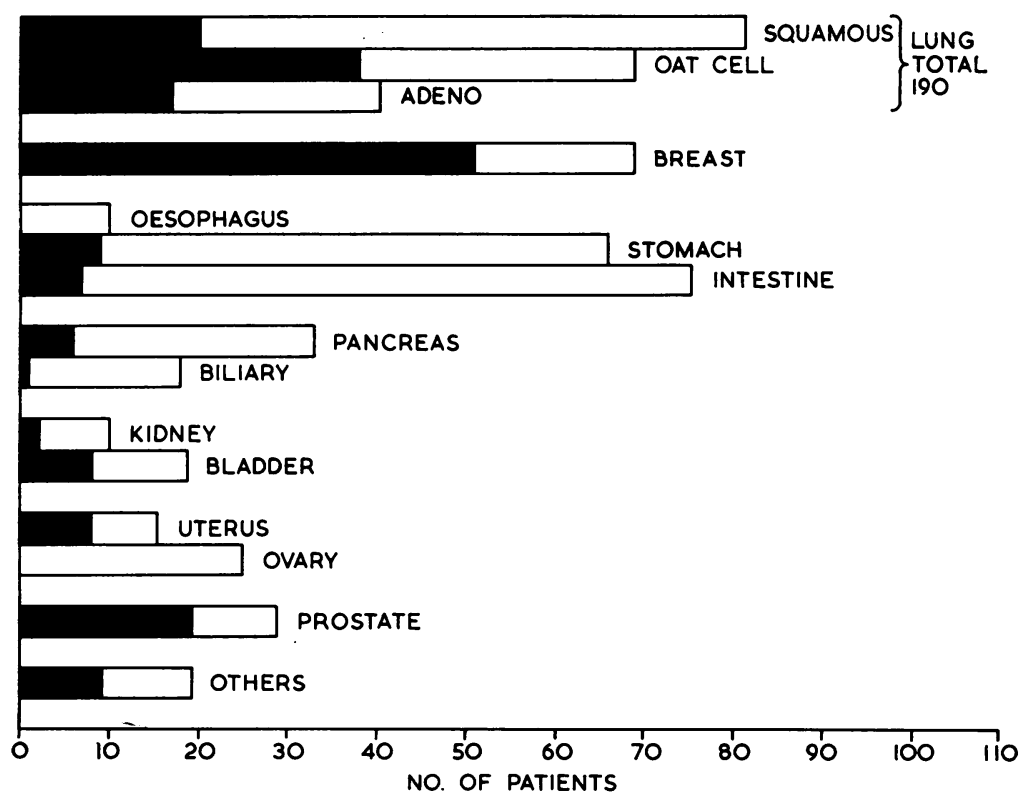

FIG. 6. The primary sites of the carcinomas. Black = patients with vertebral metastases.

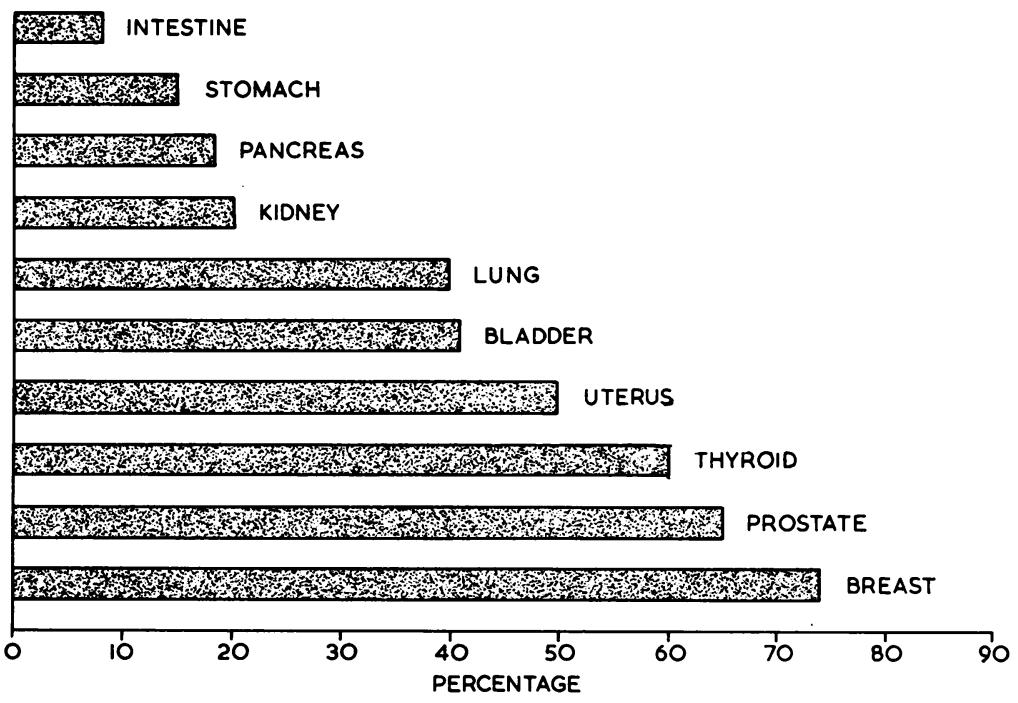

FIG. 7. The percentage incidence of vertebral metastases according to the primary site.

lung had a $40 \%$ incidence of vertebral metastasis, and although this is a high figure is shows that no one primary carcinoma is predominantly responsible for the overall findings. Tumours of which numbers were insufficient to show separately in Fig. 7 included primary carcinoma of the liver and malignant 8 melanoma of the skin (three cases each), two of each having vertebral secondaries.

The largest single group of patients, those with carcinoma of the lung, have been analysed separately. Lung carcinoma had vertebral metastases in $40 \%$, whilst all other primary sites had vertebral meta- 


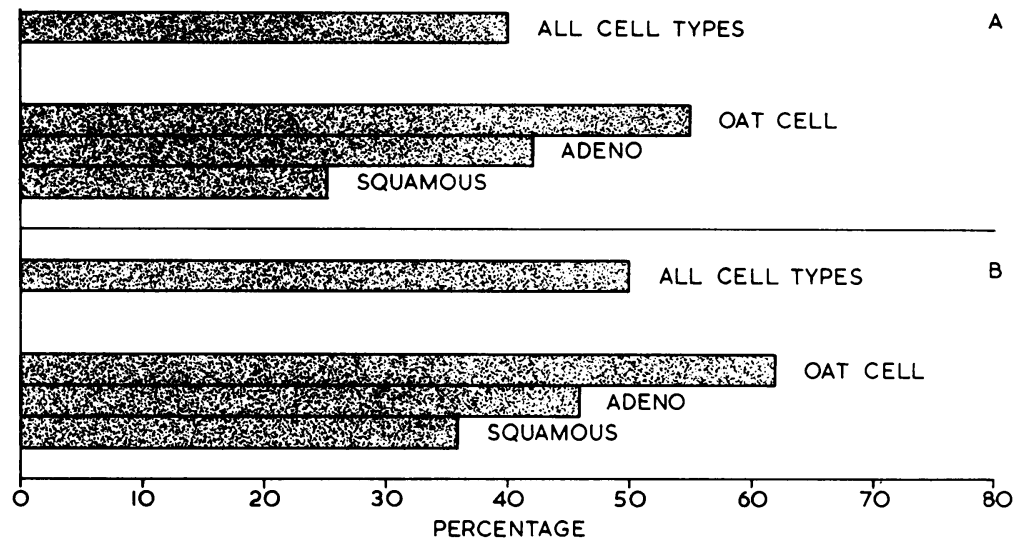

FIG. 8. Primary carcinoma of lung: percentage incidence of vertebral metastases, according to cell type. A All patients with carcinoma of lung. $B$ Patients with carcinoma of lurg with distant metastases.

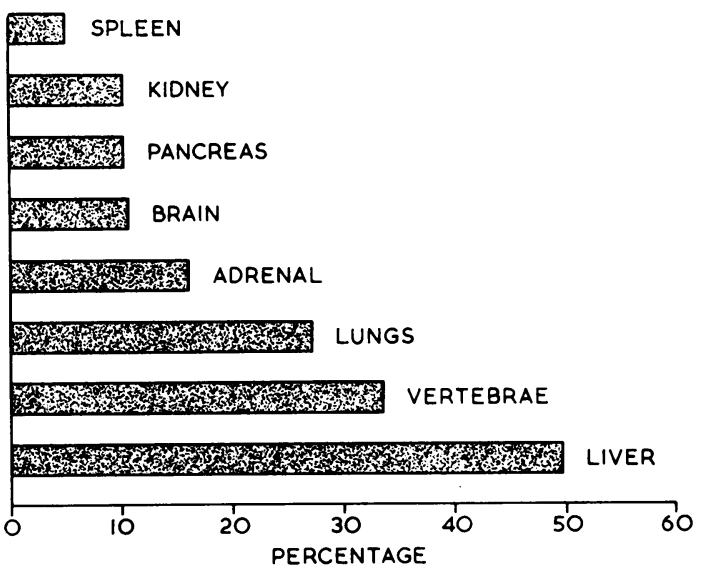

FIG. 9. All carcinomas: percentage incidence of vertebral metastases compared with metastases in other viscera.

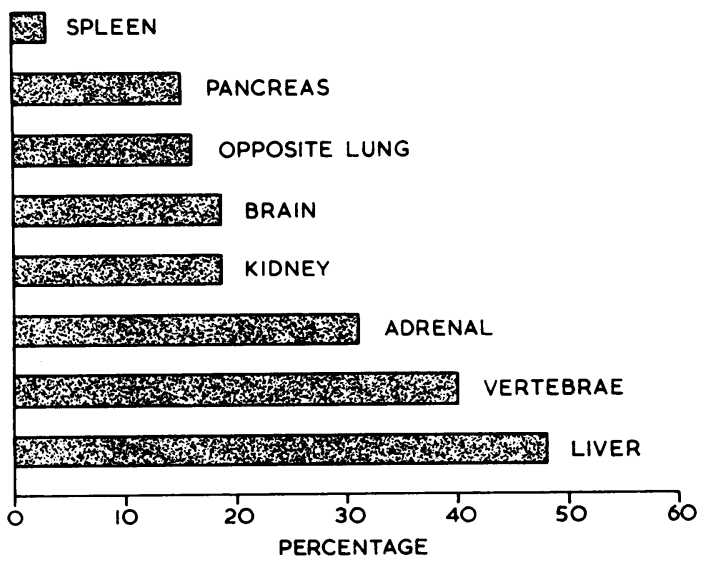

FIG. 10. Primary carcinoma of lung: percentage incidence of vertebral metastases compared with metastases in other viscera. stases in $30 \%$. Sections of the primary carcinomata of lung and their secondary tumours (an average of four tumour-bearing blocks) were reviewed and $\vec{c}$ classified in three histological groups: squamous cell carcinoma, adenocarcinoma, and oat-cell $\vec{\varphi}$ carcinoma (Fig. 6). Surgical biopsies and the cytologyos of cells in sputum were also re-examined. This: classification was less difficult than anticipated since the majority of the tumours were pure. Oat-cell carcinoma, predominantly the small cell type ando not including the large or pleomorphic cell types, $\frac{\circ}{\circ}$ was found to be clearly defined, agreeing with Azzopardi (1959) that oat-cell carcinoma was $a_{0}^{\overrightarrow{0}}$ distinctive and separate entity. The anaplastic 3 carcinomas were usually identifiable as squamous or? glandular, and the 81 squamous cell carcinomas and? the 40 adenocarcinomas both include anaplastic carcinomas. Only seven appeared to have been of genuinely mixed type, and these have been classified 3 according to their predominant or metastasizing cello type. Oat-cell carcinoma was associated with vertebral metastases $(55 \%)$ twice as frequently as $\mathrm{O}$ squamous cell carcinoma $(25 \%)$, though the larger number of localized squamous carcinomas withouto secondaries diminishes this difference when the 152 cases of carcinoma of lung with distant metastaseso were considered separately (Fig. 8).

The percentage of vertebral metastases has beenN compared with that of metastases in the other main $\omega$ viscera (Fig. 9). Vertebral metastasis $(33 \%)$ is second in frequency only to the liver $(49 \%)$, and? exceeds all other organs (lymph nodes have beenos excluded). Metastases from primary carcinoma of the lung (Fig. 10) have again been analysed separatelyô and in this group vertebral metastasis also maintainsD second place in the frequency of all secondaries. The्ष्ठ percentages of carcinomata in brain are based upon an unbroken series of 156 carcinoma patients in whom the brain was examined. 


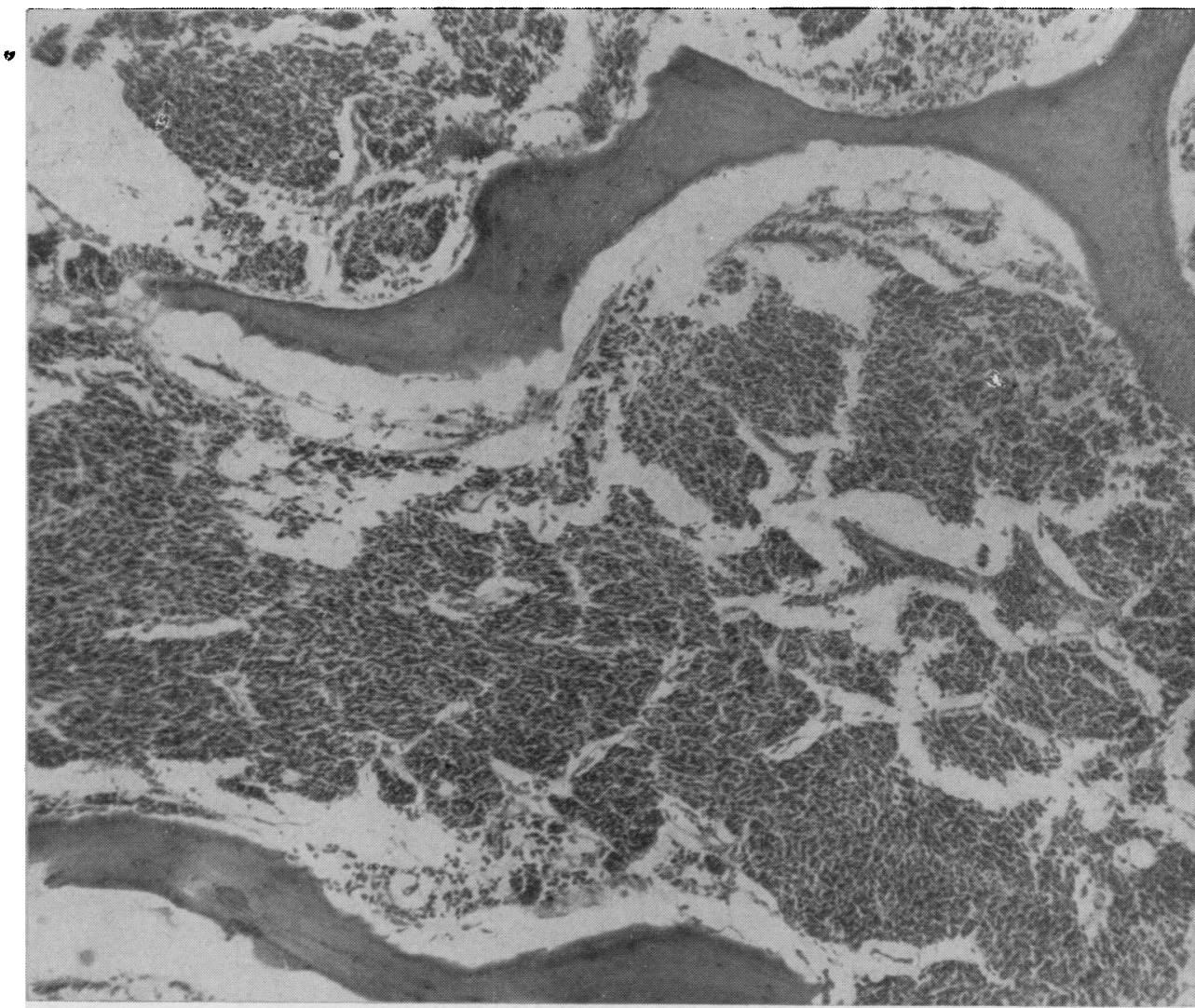

FIG. 11 .

Secondary

carcinoma in

vertebral bodies

without bony

changes.

A Oat-cell

carcinoma from

bronchus.

Haematoxylin

and eosin $\times 115$.

$B$ Cholangio-

carcinoma from

primary

carcinoma of

liver. Haema-

toxylin and

eosin $\times 115$.

A

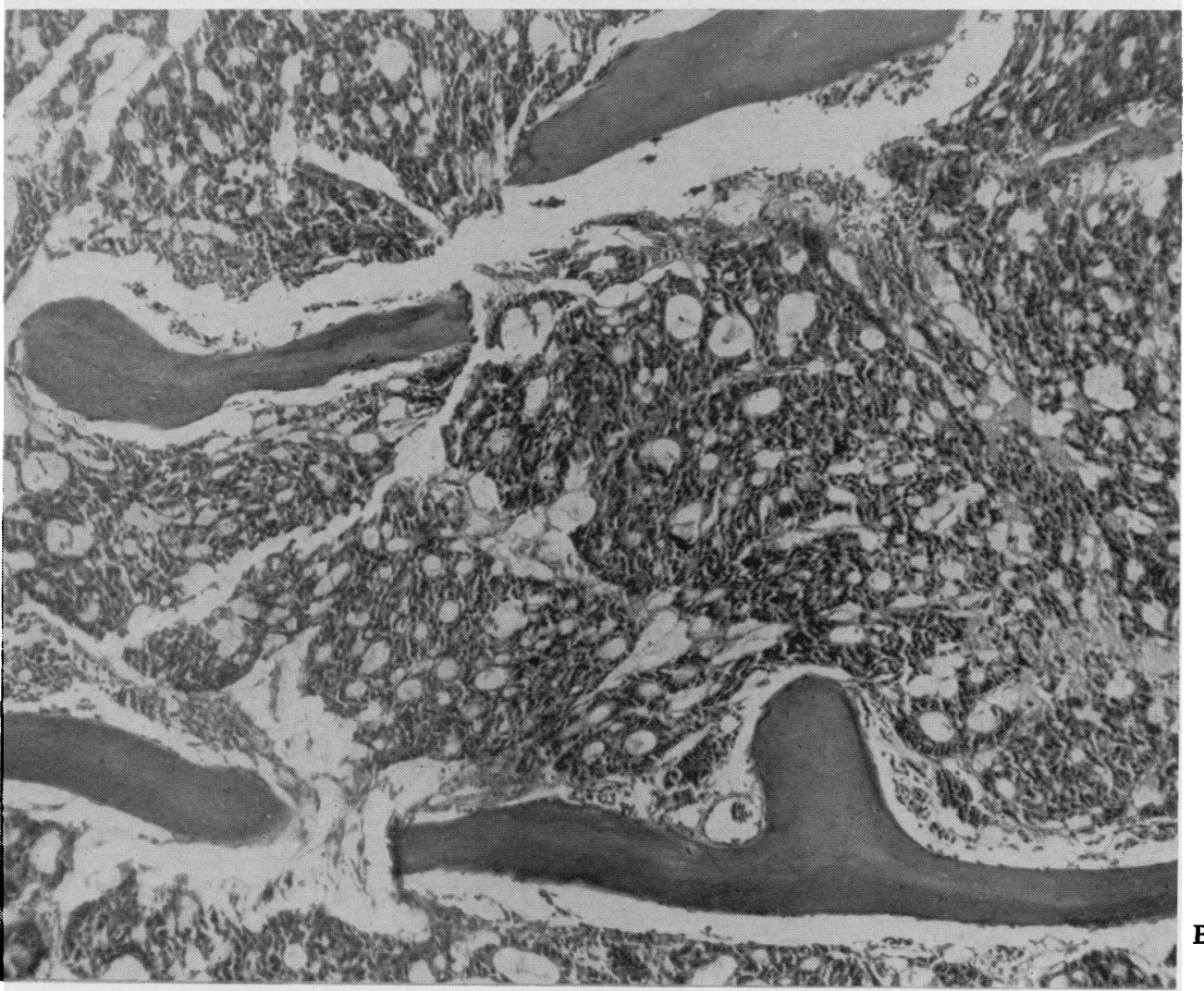


The lumbar vertebral bodies were the most common sites of a metastatic carcinoma, and the fifth lumbar vertebral body was the one with the greatest number of metastases. Dorsal vertebral bodies were frequently involved, but when they were the site of secondary carcinoma, one or more lumbar vertebrae usually also contained secondaries. Solitary dorsal vertebral metastases were found, but were rare; solitary cervical vertebral metastases were even rarer. All the cervical vertebral bodies have not been examined, as only the lower bodies were included in the slice; secondaries in these were uncommon unless there was extensive vertebral involvement by carcinoma. The ratio of the areas of the bone marrow available for direct examination in the cervical, dorsal, and lumbar bodies was $2: 5: 4$; the ratio of bone marrow volume was $2: 8: 9$. This indicates that vertebral bone marrow volume can be the cause of preferential lumbar vertebral metastasis, though we believe other factors are also involved. The site of the primary carcinoma was of importance, as elective lumbar vertebral body metastasis was seen most commonly in carcinomas arising primarily in the pelvis. If the pelvic cavity contained carcinoma, either from the uterus, prostate, or bladder, the lower lumbar vertebrae were often involved; upward spread in a centrifugal pattern did appear to take place in the vertebral bodies. Many cases of prostatic carcinoma showed such extensive vertebral carcinomatosis that the mode of spread could not be assessed, and others showed metastases scattered in a random manner, but our findings are not in accord with those of Willis (1960) who found no elective or ascending lumbar metastasis and discounted direct extension from the pelvis. Of the eight patients with vertebral metastases from carcinoma of the cervix, three had solitary involvement of L.5, and another showed carcinoma in L.4 and L.5 only. These indications of direct lymphatic spread, in contrast to haematogenous dissemination, will be discussed later.

\section{HAEMATOLOGICAL CHANGES}

Changes in the peripheral blood in patients with vertebral secondaries have been slight. Only a few of those with widespread destruction of vertebral bone marrow by carcinoma showed any evidence of leucoerythroblastic anaemia. The capacity of adult long bones with fatty marrow for taking over haemopoiesis in patients with slower growing vertebral metastases (usually from breast and prostate) appears to be sufficient to make peripheral blood changes a rarity. Moreover, in patients with extensive vertebral carcinomatosis, extramedullary haemopoiesis in the spleen and to a lesser extent in other organs was seen histologically. Anaemia and $\stackrel{\frac{*}{\overrightarrow{0}}}{\circ}$ mild leucopenia have been noted more frequently, but so many alternative explanations for these have $\underset{\vec{D}}{\vec{D}}$ been present-haemorrhage, infection, iron defici- $\overline{0}$ ency, radiotherapy, cytotoxic drugs - that it was not $\frac{C}{0}$ possible to incriminate bone marrow replacement $\frac{\rho}{\sigma}$ with any degree of certainty in many cases.

In a few untreated cases of oat-cell carcinoma, terminal purpura or frank haemorrhage have ${ }^{\text {s }}$ occurred. In these the bone marrow was slightly pale $\vec{\circ}$ or macroscopically normal, but histology showed a $\overrightarrow{\vec{\omega}}$ diffuse oat-cell carcinomatous infiltration of the ${ }_{\sigma}^{\omega}$ vertebral bone marrow, and it seems that thrombocytopenia has been the first manifestation of an acute bone marrow failure. This investigation has shown that irritative effects of metastases upon vertebral bone marrow are uncommon in comparison $\stackrel{+}{\infty}$ with the large numbers of bony metastases that have 0 been found, and that the normal compensatory mechanisms for producing bone marrow in the $c$ skeleton are sufficient in nearly all cases, excepting those with a fulminating diffuse infiltration of the $\vec{\theta}$ bone marrow with carcinoma cells.

\section{RADIOLOGICAL CHANGES}

The majority of the patients had some radiological investigations a short time before death. Most of $\frac{\otimes}{\not}$

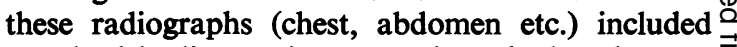
vertebral bodies, and some patients had undergone $\bar{\partial}$ specialized radiological investigation of the whole vertebral column for the demonstration of vertebral metastases. This routine radiology demonstrated a high proportion of the long-standing vertebralo metastases (usually from breast) and almost all the $\dot{0}$ vertebral carcinomata which were osteoblastic 3 . (mainly from prostate) or severely osteolytic. $\delta$ Histology showed a clear relationship between osteoblastic and sclerotic metastases and positive 0 radiology, with a less clear association between $D$ osteolytic metastases and radiological changes unless the osteolysis was gross. There remained $a_{N}$ large group with metastases (over $50 \%$ ) where radiology was negative or inadequate, and in these $N$ histology showed carcinomatous replacement of the bone marrow without significant bone change or fibrosis (Fig. 11). In these cases of vertebral metastasis without alteration of bone structure, often $\frac{\bar{\Phi}}{\complement}$ primary carcinomata of lung with recent metastases, $\stackrel{\oplus}{?}$ it cannot be expected that radiology will be reliable. 0 Young and Funk (1953), in a small series, showed a

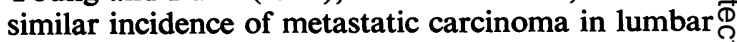
vertebral bodies $(36 \%)$ but radiological changes $\mathbb{\Phi}$ were found in less than $50 \%$ of the vertebrae $\frac{\varrho}{O}$ containing metastatic carcinoma, even when the abnormal vertebrae were removed from the bodyo 
and radiographed individually. Radiology demonstrated metastases with definite bone changes, but was negative in over half the cases with vertebral metastases; this was due to the limited bone changes that are seen in many of the secondary carcinomata. Osteoporosis and Paget's disease were often also present in the elderly adults examined, causing some false radiological positives. These findings emphasize the limitations of radiological skeletal surveys before or after death in patients suspected of metastatic carcinoma.

\section{DISCUSSION}

One of the basic aims of a necropsy is to examine as much as possible of the most rewarding parts of the body in a limited time. The vertebral slice that is removed by this technique produces for inspection about $110 \mathrm{sq} . \mathrm{cm}$. of red bone marrow from the dorsal and lumbar vertebrae. This area is similar to that seen in the bisected surfaces of both kidneys, or more than that seen in two longitudinal slices through a normal spleen. The vertebral slice produces more bone marrow than can be easily obtained from the skull, ribs, sternum, or pelvis. It is a larger area than that of the red and yellow bone marrow of the split femur, and Shillingford (1950) has re-emphasized how small is the amount of red bone marrow in the normal adult femur. The femur, though traditionally examined by pathologists, is not the best bone for the study of secondary tumours or primary disorders of adult red bone marrow.

There has been considerable disagreement in the reported overall incidence of metastatic carcinomata in the skeleton. In large series, such as those of Kitain (1922) and Willis (1952), low figures of 9 and $13.6 \%$ respectively were found. Other authors found a higher incidence; Fraenkel (1902) reported vertebral metastases in $20 \%$ and Zemgulys (1931) and Schopper (1939), reviewing a large number of German papers, both considered that metastases to the skeleton were found at necropsy in 20 to $30 \%$ of all cases of malignant disease. These variations are due largely to differences in necropsy technique, since the number of bones examined varied widely, and in many papers it was not stated which bones were examined. The older reports did not contain the large numbers of primary carcinomata of the lung that are present in this series, but it has already been shown that the complete exclusion of lung carcinoma from the present series only reduces the figure of vertebral metastasis from 33 to $30 \%$.

Even when the vertebral slice is used as the only skeletal examination, it appears to give a reasonably accurate indication of the presence of metastases in the whole of the skeletal system. Clearly no single bone can be a reliable guide to the state of another bone, but there does seem to be good evidence that the vertebral bodies are the best indication of bony metastasis. Von Recklinghausen (1891) stated that the order of frequency of metastatic carcinoma in different parts of the skeleton was as follows: vertebrae, femur, pelvis, ribs, sternum, humerus, and skull. The bony metastases analysed by Kaufmann (1902), Simpson (1926), Pürckhauer (1928), Aufses (1930), Lenz and Freid (1931), and Willis (1952), when added together, give the following numbers of metastases in the 314 patients: vertebrae 177, femur, 108, ribs 107, and skull 102. (The figures for humerus, 46 , and sternum, 34 , are not comparable as these two sites are not mentioned in all six papers.) A large radiological survey (Sutherland, Decker, and Cilley, 1932) confirmed that the vertebral bodies were common sites of metastatic tumour, but found that the pelvis was even more frequently involved. The difficulty of examining the pelvis at necropsy makes it impossible to confirm this, but it seems clear that the vertebrae are the most commonly involved parts of the skeleton that are readily accessible. No extensive comparison was made in this investigation between the incidence of metastases to the vertebral bodies and that of metastases in other bones, but the ribs and skull have been available for study, and in some cases the sternum, pelvis, or other bones have been examined. In many cases metastases in other bones were found providing the vertebral bodies were also involved, but only rarely were the vertebral bodies free from carcinoma if other skeletal metastases were present. The vertebral slice was a good screening technique for bony metastases, and the detailed examination of other bones will only add a few additional positive cases. This was further confirmed by the fact that the percentage of metastases demonstrated by the present technique has been higher than that found by other workers who investigated different parts of the skeleton (Table I).

The majority of vertebral metastases were associated with multiple systemic visceral secondaries, and could have been haematogenous. Arterial dissemination of carcinoma cells to the skeleton is theoretically likely in all the cases of primary carcinoma of the lung (190 patients) and in those with metastatic carcinoma in the lungs (106 additional patients). However, the overall incidence of metastases to the vertebral bodies was higher than that to the lungs (see Fig. 9), and in the whole series there were 53 cases with vertebral metastases which showed no macroscopic evidence of carcinoma in the lungs. Sections of the lungs were examined from all these patients, and in a few microscopic carcinomatous lymphangitis was found. It may be that more extensive histological examination of the 
TABLE I

COMPARATIVE TABLE OF BONY METASTASES

\begin{tabular}{llcl} 
Author & Primary Site & No. of Cases & Bones Examined \\
& & & \\
\hline Kaufmann (1902) & Breast & 63 & Multiple \\
Lenz and Freid (1931) & Breast & 168 & Not stated \\
Willis (1952) & Breast & 45 & Lumbar vertebral wedge and others \\
Present series & Breast & 69 & Long vertebral slice \\
Kaufmann (1902) & Prostate & 49 & Multiple \\
Pürckhauer (1928) & Prostate & 55 & Multiple \\
Present series & Prostate & 29 & Long vertebral slice \\
Galuzzi and Payne (1955) & Lung & 741 & Not stated \\
Skelton (1959) & Lung & 69 & Sternum \\
Willis (1952) & Lung & 27 & Lumbar vertebral wedge and others \\
Present s:ries & Lung & 190 & Long vertebral slice \\
Watson and Berg (1962) & Lung (oat cell) & 76 & Not stated \\
Present series & Lung (oat cell) & 69 & Long vertebral slice \\
Fraenkel (1902) & Multiple & $\ldots$ & Vertebrae \\
Kitain (1922) & Multiple & $\ldots$ & Vertebrae \\
Schopper (1939) & Multiple & $\ldots \ldots$ & Multiple \\
Skelton (1959) & Multiple & 225 & Sternum \\
Skelton (1959) & Multiple & 127 & Iliac crest \\
Present series & Multiple & 575 & Long vertebral slice
\end{tabular}

Percentage with Bone Secondaries

lungs in these paradoxical patients would have shown additional instances of microscopic pulmonary invasion, but we are satisfied that this was not present in many of these 53 patients. It is of special interest that 19 of the 53 patients with bony secondaries but without pulmonary involvement had primary pelvic carcinomas (12 prostate, four bladder, three uterus). This is further evidence in support of a lymphatic (Warren, Harris, and Graves, 1936) or venous (Batson, 1940; Henriques, 1962) pathway between the pelvis and the lumbar vertebral bodies. Our findings of predominant lumbar vertebral metastasis without pulmonary involvement in over one quarter of the 64 cases of primary carcinoma of pelvic organs (uterus, bladder, prostate) suggests that local spread via lymphatics or veins to the paravertebral plexuses is an important mode of spread to the vertebral bodies, and this has appeared to be an earlier mechanism than arterial haematogenous spread. Other cases with vertebral secondaries could have been haematogenous via the lungs but the importance of lymphatic or local venous spread in the pelvic carcinomas implies that this mechanism may play a part in other primary carcinomas. There is some evidence in our material that carcinoma of the breast has spread in this way to the dorsal vertebral bodies via the mediastinum, and the finding of pulmonary secondaries does not exclude this direct route to the vertebrae; most of the patients with carcinoma of the breast had such massive vertebral involvement that it was not possible to decide which vertebrae were invaded first. The high incidence of vertebral metastases that has been found in this study is thought to have been mainly due to the good exposure obtained by the vertebral slice technique, allowing critical examination with accurate selection of material for histology; we do not believe that all the vertebralo

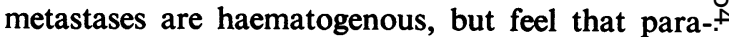
vertebral spread has contributed to the total number? of vertebrae invaded by carcinoma.

The absence of radiological changes in many vertebral bodies containing metastatic carcinoma, especially in cases of short duration, usually arising $\stackrel{\square}{\mathcal{Q}}$ from the lungs, has been emphasized in the past $\vec{F}$ (Shackman and Harrison, 1948; Young and Funk, $\frac{9}{3}$ 1953). The routine use of radiology by clinicians for the exclusion of bony metastases and the increasingo use of post-mortem radiology by pathologists has perpetuated the idea that this is an accurate and?

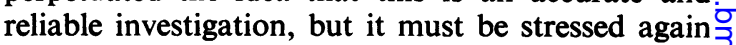
that this is not so in the case of all metastatic carcinomas. The reputation of radiology is based upon the high incidence of positive findings in primary bone tumours and certain secondary tumours, especially those from breasts and prostate. $\frac{D}{8}$ This under-diagnosis of vertebral metastasis by․ radiology has been supported by pathologists who N have been reluctant to examine the skeleton or who have done so by inadequate techniques such as the split femur or sternum or the sawn lumbar vertebral $\omega$ wedge; all these methods examine a limited amount? of red bone marrow that is obscured by bone dust. The combination of negative clinical and radiological findings with ineffective post-mortem examination ${ }^{-}$ has produced an impression that bony metastases $\frac{T}{\circ}$ are not common except from certain primary $\frac{\vec{D}}{\mathbb{D}}$ carcinomas; this study, based upon the examination $\frac{?}{\mathbb{D}}$ of the vertebral bodies and supported by histology, $\stackrel{\mathbb{Q}}{\square}$ has clearly shown that between one third and one half of all patients with metastatic carcinoma die $\delta$ with bony secondaries, and that the skeleton comeso 
second only to the liver in the order of frequency of the sites of metastases.

We are indebted to many members of the medical and technical staff of Central Middlesex Hospital, especially to our mortuary superintendent, Mr. L. G. Harvey, for his help in obtaining the vertebral slices.

\section{REFERENCES}

Aufses, A. H. (1930). Arch. Surg., 21, 916.

Azzopardi, J. G. (1959). J. Path. Bact., 78, 513.

Batson, O. V. (1940). Ann. Surg., 112, 138.

Fraenkel (1902). Münch. med. Wschr., 1, 383.

Galluzzi, S., and Payne, P. M. (1955). Brit. J. Cancer, 9, 511.

Heasman, M. A. (1962). Proc. roy. Soc. Med., 55, 733.

Henriques, C. Q. (1962). Ann. roy. Coll. Surg. Engl., 31, 1.

Kaufmann, E. (1902). Dtsch. Chir., 53, 381.

Kitain, H. (1922). Virchows Arch. path. Anat., 238, 289.

Lenz, M., and Freid, J. R. (1931). Ann. Surg., 93, 278.
Pürckhauer, R. (1928). Z. Krebsforsch., 28, 68.

Recklinghausen, F. von (1891). In Festschrift Rudolf Virchow. Reimer, Berlin.

Registrar General (1963). Statistical Review of England and Wales for 1961, p. 1. H.M.S.O., London.

Schopper, W. (1939). In Handbuch der speziellen pathologischen Anatomie und Histologie, vol. 9, p. 4, edited by O. Lubarsch and F. Henke, Springer, Berlin.

Shackman, R., and Harrison, C. V. (1948). Brit. J. Surg., 35, 385.

Shillingford, J. P. (1950), J. clin. Path., 3, 24.

Simpson, W. M. (1926). Surg. Gynec. Obstet., 42, 489.

Skelton, M. O. (1959). J. clin. Path., 12, 70.

Sutherland, C. G., Decker, F. H., and Cilley, E. I. L. (1932). Amer. J. Cancer, 16, 1457.

Warren, S., Harris, P. N., and Graves, R. C. (1936). Arch. Path., 22, 139.

Watson, W. L., and Berg, J. W. (1962). Cancer (Philad.), 15, 759.

Willis, R. A. (1952). The Spread of Tumours in the Human Body, 2nd ed. Butterworth, London.

- (1960). Pathology of Tumours, 3rd ed. Butterworth, London.

Young, J. M., and Funk, F. J. Jr. (1953). J. Bone Jt Surg., 35A, 55.

Zemgulys, J. (1931). Z. Krebsforsch., 34, 266.

\section{The May 1964 Issue}

\section{THE MAY ISSUE 1964 CONTAINS THE FOLLOWING PAPERS}

Iatrogenic endometrial patterns D. CHARLES

A histological study of hyaline deposits in laryngeal, aural, and nasal polyps and their differentiation from amyloid J. C. MCALPINE and J. D. BANCROFT

Histology of the aortic media in dissecting aneurysms GERALD MANLEY

A comparison of a fluorescent antibody technique with a cultural method in the detection of infections with Shigella sonnei C. E. D. TAYLOR, G. V. HEIMER, D. J. LEA, and A. J. H. TOMLINSON

A new selective blood agar medium for Streptococcus pyogenes and other haemolytic streptococci E. J. L. LOWBURY, A. KIDSON, and H. A. LILLY

Pyocine-typing of hospital strains of Pseudomonas pyocyanea J. H. DARRELL and A. H. WAHBA

The evolution of new hospital strains of Staphylococcus aureus M. PATRICIA JEVONS and M. T. PARKER

Subcutaneous infection due to Aspergillus terreus H. D. CHEETHAM

The survival of Haemophilus influenzae and pneumococci in specimens of sputum sent to the laboratory by post J. ROBERT MAY and DOREEN M. DELVES

Changes in serum and urinary potassium levels during profound hypothermia in man L. LANGDON and D. P. E. KINGSLEY

The microdetermination of biological copper with oxalyldihydrazide R. N. BEALE and D. CROFT
Auto-immunity to prostate antigen in rheumatic disease A. GRIMBLE

A micro-gel precipitin reaction for Hashimoto's thyroiditis M. GOLDIN and A. GLENN

The estimation of uranin (fluorescein sodium) in blood T. WAGATSUMA and HELEN PAYLING WRIGHT

Oligosaccharides in infants' faeces S. BAAR and H. BICKEL A comparison of platelet aggregation produced by seven compounds and a comparison of their inhibitors J. R. O'BRIEN

The effect of calcium on fibrinolysis in vitro SALLY BRUCE The laboratory control of thrombolytic therapy CHRISTINE HAWKEY and MARGARET HOWELL

The effect of age on normal values of the Westergren sedimentation rate F. M. HILDER and F. W. GUNZ

Measurement of antibodies reacting with capsular antigens of Haemophilus influenzae D. C. TURK and C. A. GREEN

Short-term storage of Haemophilus influenzae $\quad$ D. C. TURK

Technical methods

A shortened automated procedure for the determination of alkaline phosphatase JOYCE L. BELL and M. COLLIER with the technical assistance of ANNA MARIE HARTMANN

Book reviews

Second fibrinolysis workshop

Copies are still available and may be obtained from the PUBLISHING MANAGER, BRITISH MEDICAL ASSOCIATION, TAVISTOCK SQUARE, W.C.I., price 18s. 6D. 